\title{
RIBAUCOUR TRANSFORMATIONS ON LORENTZIAN SPACE FORMS IN LORENTZIAN SPACE FORMS
}

\author{
JOONSANG PARK
}

\begin{abstract}
We study Ribaucour transformations on nondegenerate local isometric immersions of Lorentzian space forms into Lorentzian space forms with the same sectional curvatures which have flat normal bundles. They can be associated to dressing actions on the solution space of Lorentzian Grassmannian systems.
\end{abstract}

\section{Introduction}

In this paper, we consider Ribaucour transformations of local nondegenerate isometric immersions of Lorentzian space forms $N^{n, 1}(c)$ into Lorentzian space forms $N^{n+k, 1}(c)$ which has flat normal bundles. Classically, a Ribaucour transformation on a surface in $\mathbb{R}^{3}$ is defined as a sphere congruence which preserves principal directions, and it is applied to special kinds of surfaces such as surfaces with constant mean curvature, surfaces with constant Gaussian curvature and so on [3]. The definition of a Ribaucour transformation is generalized and studied to higher dimensional submanifolds in space forms $[1,2]$. In particular, Dajczer and Tojeiro treated Ribaucour transformations of Riemannian submanifolds in pseudo-Riemannian space form [2].

On the other hand, many interesting submanifolds have special Gauss-Codazzi equations in that they are solution equations $[5,6,7,8,9]$. In the theory of integrable system, there is a systematic method called a dressing action to produce a new solution from a known solution. The problem of immersions of space forms into space forms and their transformations can be explained by means of the theory of integrable system, too. In this vein, immersions of Riemannian space form $N^{n}(c)$ into Riemannian space form $N^{n+k}(c)$ can be associated to the solutions of Grassmannian system and some dressing actions on them are constructed in [1], and immersions of Lorentzian space form $N^{n, 1}(c)$ into Lorentzian space form $N^{n+k, 1}(c)$ are associated to the solutions of Lorentzian Grassmannian system [4].

Received December 26, 2006; Revised March 3, 2008.

2000 Mathematics Subject Classification. 57N35.

Key words and phrases. Lorentzian space forms, Ribaucour transformation, flat connection, nondegenerate, Lorentzian Grassmannian system.

This work was supported by the research program of Dongguk University.

(C)2008 The Korean Mathematical Society 
The goal of this paper is to find all the local Ribaucour transformations of nondegenerate isometric immersions of $N^{n, 1}(c)$ into $N^{n+k, 1}(c)$ which has flat normal bundles, to construct dressing actions on the solution space of the Lorentzian Grassmannian system and finally to show that these actions are all the Ribaucour transformations on these immersions.

\section{Lorentzian space forms in Lorentzian space form}

We denote by $\mathbb{R}^{n, r}$ the vector space $\mathbb{R}^{n+r}$ with the nondegenerate inner product of index $r$,

$$
\langle x, y\rangle=-\sum_{i=1}^{r} x_{i} y_{i}+\sum_{i=r+1}^{n+r} x_{i} y_{i}
$$

The simply connected complete $(m+1)$-dimensional Lorentzian manifold $N^{m, 1}(c)$ of the constant sectional curvature $c=0,1$ or -1 is locally isometric to the Lorentzian space $\mathbb{R}^{m, 1}$, the Lorentzian sphere $\mathbb{S}^{m, 1}$, or the Lorentzian hyperbolic space $\mathbb{H}^{m, 1}$, respectively, where

$$
\begin{aligned}
& \mathbb{S}^{m, 1}=\left\{x \in \mathbb{R}^{m+1,1} \mid\langle x, x\rangle=1\right\}, \\
& \mathbb{H}^{m, 1}=\left\{x \in \mathbb{R}^{m, 2} \mid\langle x, x\rangle=-1\right\} .
\end{aligned}
$$

Suppose $X: M^{n, 1} \longrightarrow N^{n+k, 1}(c)$ is an isometric immersion of an $(n+1)$ dimensional Lorentzian manifold $M^{n, 1}$ in the $(n+k+1)$-dimensional Lorentzian space form $N^{n+k, 1}(c)$.

Definition 2.1. $M^{n, 1}$ is called nondegenerate if the image of the second fundamental form $\operatorname{Im} I I_{p}=\left\{I I_{p}(Y, Z) \mid Y, Z \in T_{p} M\right\}$ has dimension $n+1$ for any $p \in M^{n, 1}$.

If the normal bundle is flat, then it is an elementary fact that all the shape operators $\left\{A_{v} \mid v \in T_{p}^{\perp} N^{n, 1}(c)\right\}$ commute for any $p \in M$. Even though self-adjoint operators on the Lorentzian vector space are not diagonalizable in general, it holds in the following case.

Lemma $2.2([4])$. Suppose $X: N^{n, 1}(c) \longrightarrow N^{n+k, 1}(c)$ is a local nondegenerate isometric immersion and the normal bundle is flat. Then all the shape operators are simultaneously diagonalizable.

Hence, for such $M$, it makes sense to say principal directions and it turns out to be true that $M$ has a principal coordinate system. More precisely,

Proposition 2.3 ([4]). Let $X: N^{n, 1}(c) \longrightarrow N^{n+k, 1}(c)$ be a nondegenerate isometric immersion with a flat normal bundle, and assume $k \geq n+1$. Then, for a local parallel normal frame $e_{\alpha}(n+1 \leq \alpha \leq n+k+1)$, there exist a curvature coordinate system $\left(x_{1}, \ldots, x_{n+1}\right)$, a map $b=\left(b_{1}, \ldots, b_{n+1}\right)^{t}$ and an $(n+1) \times k$ matrix-valued map $B_{1}=\left(b_{i j}\right)$ such that $e_{i}=\frac{1}{b_{i}} \frac{\partial}{\partial x_{i}}$ are a principal 
tangent frame, $B_{1} B_{1}^{t}=I_{n+1}$ and the first and second fundamental forms are given by

$$
I=\sum_{i=1}^{n+1} \epsilon_{i} b_{i}^{2} d x_{i}^{2}, \quad I I=\sum_{i=1}^{n+1} \sum_{r=1}^{k} \epsilon_{i} b_{i r} b_{i} d x_{i}^{2} \otimes e_{n+1+r}
$$

where $I_{m}$ is the $m \times m$ identity matrix and $\epsilon_{i}=\left\langle e_{i}, e_{i}\right\rangle$.

The local geometry of the immersion $X$ in Proposition 2.3 can be described by $(2.1)$ as follows. For a map $f$, denote $f_{x_{i}}=\frac{\partial f}{\partial x_{i}}$. Let $\bar{\nabla}$ be the Levi-Civita connection for $N^{n+k, 1}(c)$ induced by the usual differential $d$. Put $f_{i j}=\frac{\left(b_{i}\right)_{x_{j}}}{b_{j}}$ for $i \neq j$. Then the Levi-Civita connection 1-form $\omega=\left(\omega_{i j}\right)$ on $M$ and the shape operator $A_{e_{\alpha}}=\sum_{i=1}^{n+1} e_{i} \otimes \omega_{i \alpha}$ satisfy

$$
\left\{\begin{array}{l}
\omega_{i j}=f_{i j} d x_{i}-\epsilon_{i} \epsilon_{j} f_{j i} d x_{j}, \\
\omega_{i, n+1+r}=b_{i r} d x_{i}, \\
\left(f_{i j}\right)_{x_{k}}=f_{i k} f_{k j} \text { for distinct } i, j, k, \\
\left(f_{i j}\right)_{x_{j}}+\epsilon_{i} \epsilon_{j}\left(f_{j i}\right)_{x_{i}}+\epsilon_{j} \sum_{k} \epsilon_{k} f_{i k} f_{j k}+c \epsilon_{j} b_{i} b_{j} \quad \text { for } i \neq j, \\
\left(b_{i r}\right)_{x_{j}}=f_{i j} b_{j r} \text { for } i \neq j .
\end{array}\right.
$$

Throughout this paper, we will always assume that $-\epsilon_{1}=\epsilon_{2}=\cdots=\epsilon_{n+1}$, i.e., $e_{1}$ is a timelike direction. It is convenient to use matrix notations for $(2.2)$. Let $J=\operatorname{diag}(-1,1, \ldots, 1), \delta=\operatorname{diag}\left(d x_{1}, \ldots, d x_{n+1}\right), \omega=\left(\omega_{i j}\right)$ and $F=\left(f_{i j}\right)$, where $f_{i i}=0$. Then $(2.2)$ becomes

$$
\left\{\begin{array}{l}
\omega=\delta F-J F^{t} \delta J, \\
\delta \wedge d b=\omega \wedge \delta b, \\
d \omega+\omega \wedge \omega=c \delta b \wedge b^{t} \delta J, \\
\delta \wedge d B_{1}=\omega \wedge \delta B_{1} .
\end{array}\right.
$$

\section{Ribaucour transformations}

In this section, we investigate on Ribaucour transformations of isometric immersions $X: M \longrightarrow N^{m, 1}(c)$. Classically, a Ribaucour transformation is defined [3] between surfaces in the 3-dimensional Euclidean space as a sphere congruence which preserves principal directions. This definition can be generalized to submanifolds in Riemannian or Lorentzian space forms $[1,2]$.

Definition 3.1. Let $X: M \longrightarrow N^{m, 1}(c)$ and $\tilde{X}: \tilde{M} \longrightarrow N^{m, 1}(c)$ be isometric immersions. A diffeomorphism $\ell: M \longrightarrow \tilde{M}$ is called a Ribaucour transformation if $X(p) \neq \tilde{X}(\ell(p))$ for any $p \in M$ and there exists a bundle isometry $\wp: T^{\perp} M \longrightarrow T^{\perp} \tilde{M}$ covering $\ell: M \longrightarrow \tilde{M}$ such that

(1) the geodesic in direction $\xi_{p} \in T_{p}^{\perp} M$ at $p \in M$ and $\wp\left(\xi_{p}\right) \in T_{\ell(p)}^{\perp} \tilde{M}$ at $\ell(p) \in \tilde{M}$ intersects at a point equidistant to $p$ and $\ell(p)$, 
(2) if $e_{p}$ is an eigenvector of the shape operator $A_{\xi}$ of $M$, then $\ell_{*}\left(e_{p}\right)$ is an eigenvector of $A_{\wp(\xi)}$, and the geodesics in these directions intersect at a point equidistant to $p$ and $\ell(p)$.

Suppose $X: N^{n, 1}(c) \longrightarrow N^{n+k, 1}(c)$ and $\tilde{X}: N^{n, 1}(c) \longrightarrow N^{n+k, 1}(c)$ are nondegenerate isometric immersions with flat normal bundles, which are in a Ribaucour transformation. Let $e_{i}$ and $\tilde{e_{i}}$ are local principal tangent frames, $e_{\alpha}$ and $\tilde{e}_{\alpha}$ are local parallel normal frames for $X$ and $\tilde{X}$, respectively.

Put $\wp(X)=\tilde{X}$ only when $c \neq 0$, and $\wp\left(e_{i}\right)=\tilde{e_{i}}, \wp\left(e_{\alpha}\right)=\tilde{e}_{\alpha}$. Then the isometry $\wp$ can be extended to an isometry $\wp: X^{*} T V \longrightarrow \tilde{X}^{*} T V$, where $V$ is $\mathbb{R}^{m, 1}, \mathbb{R}^{m+1,1}, \mathbb{R}^{m, 2}$ for $c=0,1,-1$, respectively. According to the conditions (1) and (2) in the Definition 3.1, there exists $\zeta \in V$ such that

$$
\wp(v)-v=\langle\zeta, v\rangle(X-\tilde{X})
$$

for any $v \in V$. Take a nowhere vanishing function $\mu$ and a vector field $\eta$ with $\|\eta\|^{2}=\epsilon= \pm 1$ such that

$$
\tilde{X}=X+2 \mu \eta \text {. }
$$

Put $\eta=c \eta_{0} X+\sum_{i} \epsilon_{i} \eta_{i} e_{i}+\sum_{\alpha} \eta_{\alpha} e_{\alpha}$, where $\eta_{i}=\left\langle e_{i}, \eta\right\rangle$ and $\eta_{\alpha}=\left\langle e_{\alpha}, \eta\right\rangle$. Then by (3.1), we can prove by a direct calculation that

$$
\begin{gathered}
\eta_{0}=-\epsilon \mu, \\
\tilde{e}_{A}=e_{A}-2 \epsilon \mu \eta_{A} \eta, \quad 1 \leq A \leq n+k+1 .
\end{gathered}
$$

As was shown for Ribaucour transformations on Riemannian submanifolds in pseudo-Riemannian space form [2], we can prove the following theorem.

Theorem 3.2. Suppose $\ell$ is a local Ribaucour transformation between nondegenerate isometric immersions $X, \tilde{X}: N^{n, 1}(c) \longrightarrow N^{n+k, 1}(c)$ with flat normal bundles, and $b_{i}, b_{i \alpha}$ and $\tilde{b}_{i}, \tilde{b}_{i \alpha}$ are the coefficients of fundamental forms for $X$ and $\tilde{X}$. Then

$$
\tilde{X}=X-2 \nu \gamma_{0}\left(c \gamma_{0} X+\sum_{i} \epsilon_{i} \gamma_{i} e_{i}+\sum_{r} \beta_{r} e_{n+1+r}\right),
$$

where $\nu^{-1}=c \gamma_{0}^{2}+\sum_{i} \epsilon_{i} \gamma_{i}^{2}+\sum_{r} \beta_{r}^{2}$, and $\gamma_{0}, \gamma_{i}, \beta_{r}$ satisfy

$$
\begin{aligned}
& \left(\gamma_{0}\right)_{x_{i}}=b_{i} \gamma_{i}, \\
& \left(\gamma_{j}\right)_{x_{i}}=\gamma_{i} f_{i j}, \text { for } i \neq j, \\
& \left(\beta_{r}\right)_{x_{i}}=\gamma_{i} b_{i r},
\end{aligned}
$$

where $f_{i j}=\left(b_{i}\right)_{x_{j}} / b_{j}$. The equations (3.6) are completely integrable. Moreover,

$$
\begin{gathered}
\tilde{b}_{i}=b_{i}-2 \nu \gamma_{0} \Gamma_{i}, \\
\tilde{b}_{i r}=b_{i r}-2 \nu \beta_{r} \Gamma_{i},
\end{gathered}
$$

where $\Gamma_{i}=c b_{i} \gamma_{0}+\epsilon_{i}\left(\gamma_{i}\right)_{x_{i}}+\sum_{j \neq i} \epsilon_{j} f_{i j} \gamma_{j}+\sum_{r} b_{i r} \beta_{r}$. 
Proof. Since $\tilde{X}_{x_{i}}$ is parallel to $\tilde{e}_{i}$, we have $\left\langle d \tilde{X}\left(e_{i}\right), \tilde{e}_{A}\right\rangle=0$ for $A \neq i$. Thus from (3.4), we get

$$
\eta_{A} d \mu\left(e_{i}\right)=-\epsilon \eta_{A} \eta_{i}+\mu\left[d \eta_{A}\left(e_{i}\right)-\left\langle\bar{\nabla}_{e_{i}} e_{A}, \eta\right\rangle\right]
$$

Put $g_{i}=\left[d \mu\left(e_{i}\right)+\epsilon \eta_{i}\right] / \mu$, then (3.9) implies

$$
\begin{gathered}
d \mu\left(e_{i}\right)=g_{i} \mu-\epsilon \eta_{i} \\
d \eta_{A}\left(e_{i}\right)=g_{i} \eta_{A}+\left\langle\bar{\nabla}_{e_{i}} e_{A}, \eta\right\rangle .
\end{gathered}
$$

A direct calculation for $\left\langle\bar{\nabla}_{e_{i}} e_{A}, \eta\right\rangle$ using (2.2) shows that

$$
\begin{aligned}
& d \eta_{j}\left(e_{i}\right)=g_{i} \eta_{j}+\frac{\eta_{i} f_{i j}}{b_{i}}, \quad(i \neq j \leq n+1) \\
& d \eta_{n+1+r}\left(e_{i}\right)=g_{i} \eta_{n+1+r}+\frac{\eta_{i} b_{i r}}{b_{i}} .
\end{aligned}
$$

Now, we claim that the 1 -form $\psi=\sum_{i} \epsilon_{i} g_{i} \omega_{i}$ is closed, where $\omega_{i}$ is the dual 1 -form of $e_{i}$, that is, $\omega_{i}\left(e_{j}\right)=\left\langle e_{i}, e_{j}\right\rangle=\epsilon_{i} \delta_{i j}$. To show this, using (3.10), (3.11), and (2.2), we have

$$
\begin{aligned}
\epsilon d \psi= & \epsilon d(d \mu / \mu)+d\left(\frac{1}{\mu} \sum_{i} \epsilon_{i} \eta_{i} \omega_{i}\right) \\
= & -\frac{d \mu}{\mu^{2}} \wedge \sum_{i} \epsilon_{i} \eta_{i} \omega_{i}+\frac{1}{\mu} \sum_{i} \epsilon_{i} d \eta_{i} \wedge \omega_{i}+\frac{1}{\mu} \sum_{i} \epsilon_{i} \eta_{i} d \omega_{i} \\
= & -\frac{1}{\mu^{2}}\left(\mu \psi-\epsilon \sum_{j} \epsilon_{j} \eta_{j} \omega_{j}\right) \wedge \sum_{i} \epsilon_{i} \eta_{i} \omega_{i} \\
& +\frac{1}{\mu}\left(\psi \wedge \sum_{j} \epsilon_{j} \eta_{j} \omega_{j}+\sum_{j, k} \epsilon_{k} \eta_{k} \omega_{j k} \wedge \omega_{j}\right)+\frac{1}{\mu} \sum_{i} \epsilon_{i} \eta_{i} d \omega_{i} \\
= & 0 .
\end{aligned}
$$

Thus $\psi=-d h / h$ for some $h$. Put $\gamma_{0}=-\epsilon h \mu, \gamma_{i}=h \eta_{i}$ and $\beta_{r}=h \eta_{n+r+1}$. Then (3.11) becomes the desired equations (3.6) and $\tilde{X}, \tilde{e}_{i}$ and $\tilde{e}_{\alpha}$ are of the form

$$
\begin{aligned}
& \tilde{X}=X-2 \nu \gamma_{0} w, \\
& \tilde{e}_{i}=e_{i}-2 \nu \gamma_{i} w \\
& \tilde{e}_{n+1+r}=e_{n+1+r}-2 \nu \beta_{r} w,
\end{aligned}
$$

where $w=c \gamma_{0} X+\sum_{i} \epsilon_{i} \gamma_{i} e_{i}+\sum_{r} \beta_{r} e_{n+1+r}, \nu^{-1}=\|w\|^{2}=c \gamma_{0}^{2}+\sum_{i} \epsilon_{i} \gamma_{i}^{2}+$ $\sum_{r} \beta_{r}^{2}$. Now, a tedious calculation shows that $\left\langle\tilde{X}_{x_{i}}, \tilde{e}_{j}\right\rangle=\left\langle\tilde{X}_{x_{i}}, \tilde{e}_{\alpha}\right\rangle=0$ for $i \neq j$ and $\alpha>n+1$, and thus

$$
\tilde{X}_{x_{i}}=\tilde{b}_{i} \tilde{e}_{i} .
$$


To find $\tilde{b}_{i}$, compute

$$
\begin{aligned}
\left\langle\tilde{X}_{x_{i}}, \tilde{e}_{i}\right\rangle= & \left\langle b_{i} e_{i}-2\left(\nu \gamma_{0}\right)_{x_{i}} w-2 \nu \gamma_{0} w_{x_{i}}, e_{i}-2 \nu \gamma_{i} w\right\rangle \\
= & \epsilon_{i} b_{i}-2\left(\nu \gamma_{0}\right)_{x_{i}} \gamma_{i}-2 \nu \gamma_{0}\left[\left(\gamma_{i}\right)_{x_{i}}-\left\langle\bar{\nabla}_{X_{x_{i}}} e_{i}, w\right\rangle\right] \\
& \quad-2 \nu b_{i} \gamma_{i}^{2}+4\left(\nu \gamma_{0}\right)_{x_{i}} \gamma_{i}+2 \nu^{2} \gamma_{0} \gamma_{i}(1 / \nu)_{x_{i}} \\
= & \epsilon_{i} b_{i}-2 \nu \gamma_{0}\left[\left(\gamma_{i}\right)_{x_{i}}-\left\langle\bar{\nabla}_{X_{x_{i}}} e_{i}, w\right\rangle\right] \\
= & \epsilon_{i} b_{i}-2 \epsilon_{i} \nu \gamma_{0}\left[\epsilon_{i}\left(\gamma_{i}\right)_{x_{i}}+c b_{i} \gamma_{0}+\sum_{j \neq i} \epsilon_{j} f_{i j} \gamma_{j}+\sum_{r} b_{i r} \beta_{r}\right] \\
= & \epsilon_{i}\left(b_{i}-2 \nu \gamma_{0} \Gamma_{i}\right) .
\end{aligned}
$$

Hence we get $\tilde{b}_{i}=b_{i}-2 \nu \gamma_{0} \Gamma_{i}$. A similar argument, which we will omit, shows that

$$
\bar{\nabla}_{X_{x_{i}}} \tilde{e}_{n+1+r}=\tilde{b}_{i r} \tilde{e}_{i},
$$

where $\tilde{b}_{i r}=b_{i r}-2 \nu \beta_{r} \Gamma_{i}$.

Put

$$
\gamma=c \gamma_{0} X+\sum_{i} \epsilon_{i} \gamma_{i} e_{i} \text { and } \beta=\sum_{r} \beta_{r} e_{n+1+r}
$$

and let

$$
\|\gamma\|_{n+1, c}^{2}=c \gamma_{0}^{2}+\sum_{i} \epsilon_{i} \gamma_{i}^{2} \quad \text { and } \quad\|\beta\|^{2}=\sum_{r} \beta_{r}^{2} .
$$

We will say that $X, \tilde{X}$ are in a Ribaucour transformation in tangentially spacelike direction if $\|\gamma\|_{n+1, c}^{2}>0$, and tangentially timelike direction if $\|\gamma\|_{n+1, c}^{2}<0$.

If $\Gamma_{i}=0$ for all $i$, then $X$ and $\tilde{X}$ are congruent by (3.7) and (3.8), which is not an interesting case.

Theorem 3.3. If $\Gamma_{i} \neq 0$ in Theorem 3.2, then $\nu\|\beta\|^{2}=C$ for some constant $C$. Hence when $\ell$ is tangentially spacelike,

$$
\tilde{X}=X-\frac{2 \sin \rho \gamma_{0}}{\|\beta\|^{2}}\left(c \gamma_{0} X+\sum_{i} \epsilon_{i} \gamma_{i} e_{i}+\sum_{r} \beta_{r} e_{n+1+r}\right),
$$

and when it is tangentially timelike,

$$
\tilde{X}=X-\frac{2 \cosh \rho \gamma_{0}}{\|\beta\|^{2}}\left(c \gamma_{0} X+\sum_{i} \epsilon_{i} \gamma_{i} e_{i}+\sum_{r} \beta_{r} e_{n+1+r}\right)
$$

or

$$
\tilde{X}=X+\frac{2 \sinh \rho \gamma_{0}}{\|\beta\|^{2}}\left(c \gamma_{0} X+\sum_{i} \epsilon_{i} \gamma_{i} e_{i}+\sum_{r} \beta_{r} e_{n+1+r}\right)
$$

for some constant $\rho$. 
Proof. From (3.12), we know that $\tilde{b}_{i r}$ are the coefficients of the second fundamental form of $\tilde{X}$ and thus

$$
1=\sum_{r} \tilde{b}_{i r}^{2}=\sum_{r}\left(b_{i r}-2 \nu \beta_{r} \Gamma_{i}\right)^{2}=1-4 \nu \sum_{r} \beta_{r} b_{i r} \Gamma_{i}+4 \nu^{2}\|\beta\|^{2} \Gamma_{i}^{2}
$$

so that

$$
\|\beta\|^{2} \Gamma_{i}=\left(\|\gamma\|_{n+1, c}^{2}+\|\beta\|^{2}\right) \sum_{r} \beta_{r} b_{i r}
$$

Hence

$$
\begin{aligned}
& \left(\|\gamma\|_{n+1, c}^{2}+\|\beta\|^{2}\right)^{2}\left(\nu\|\beta\|^{2}\right)_{x_{i}} \\
= & \left(\|\beta\|^{2}\right)_{x_{i}}\left(\|\gamma\|_{n+1, c}^{2}+\|\beta\|^{2}\right)-\|\beta\|^{2}\left(\|\gamma\|_{n+1, c}^{2}+\|\beta\|^{2}\right)_{x_{i}} \\
= & \left(\|\beta\|^{2}\right)_{x_{i}}\|\gamma\|_{n+1, c}^{2}-\|\beta\|^{2}\left(\|\gamma\|_{n+1, c}^{2}\right)_{x_{i}} \\
= & 2\|\gamma\|_{n+1, c}^{2} \sum_{r} \beta_{r} b_{i r} \gamma_{i}-2\|\beta\|^{2}\left[c b_{i} \gamma_{0} \gamma_{i}+\sum_{j \neq i} \epsilon_{j} f_{i j} \gamma_{j} \gamma_{i}+\epsilon_{i}\left(\gamma_{i}\right)_{x_{i}} \gamma_{i}\right] \\
= & 2 \gamma_{i}\left[\|\beta\|^{2} \Gamma_{i}-\left(\|\gamma\|_{n+1, c}^{2}+\|\beta\|^{2}\right) \sum_{r} \beta_{r} b_{i r}\right] \\
= & 0 .
\end{aligned}
$$

Therefore, $\nu\|\beta\|^{2}$ is constant.

\section{Lorentzian Grassmannian systems and dressing actions}

In [4], the nondegenerate local isometric immersions

$$
X: N^{n, 1}(c) \longrightarrow N^{n+k, 1}(c)
$$

with flat normal bundles are associated to the solutions of the so-called Lorentzian Grassmannian system. The Lorentzian Grassmannian system is a kind of $G / K$ system, which is developed by Terng [9]. We will first briefly review the Lorentzian Grassmannian system and summarize how to relate such immersions with the Lorentzian Grassmannian system, and next construct two kind of actions on the solution space of the system to produce a new such immersion. These actions will turn out to be all the Ribaucour transformations for $X$.

Let $\mathcal{M}_{p \times q}$ be the set of $p \times q$ matrices, $\mathbb{R}^{m}=\mathcal{M}_{m \times 1}, J=\operatorname{diag}(-1,1, \ldots, 1)$, $J_{c}=\operatorname{diag}(c,-1,1, \ldots, 1)$. Denote $u=\left(u_{0}, u_{1}, \ldots, u_{m}\right)^{t} \in \mathbb{R}^{m+1}$. For $c=0,1$ or -1 , the isometry group $G_{c}$ of $N^{n+k, 1}(c)$ is

$$
G_{c}=\left\{A \in G L(n+k+2, \mathbb{R}) \mid A J_{c} A^{t}=J_{c}\right\},
$$

and the Lie algebra of $G_{c}$ is

$$
\mathcal{G}_{c}=\left\{\left(\begin{array}{cc}
0 & -c \xi^{t} J \\
\xi & Y
\end{array}\right) \mid Y \in o(n+k, 1), \xi \in \mathbb{R}^{n+k+1}\right\} .
$$

Note that when $c=0$, the first column $( \pm 1, X)$ of $A \in G_{c}$ can be identified with $X \in \mathbb{R}^{m, 1}$ by $X \leftrightarrow( \pm 1, X)$. 
Definition 4.1. Let $k \geq n+1$. The Lorentzian Grassmannian system is a partial differential equation for $(b, F, G) \in \mathcal{M}_{(n+1) \times 1} \times \mathcal{M}_{(n+1) \times(n+1)} \times$ $\mathcal{M}_{(k-n-1) \times(n+1)}$ with $f_{i i}=0$ for $F=\left(f_{i j}\right)$, such that the 1-parameter family of connection 1 -form

$$
\theta_{\lambda}=\left(\begin{array}{cccc}
0 & -c b^{t} \delta J & 0 & 0 \\
\delta b & \delta F-J F^{t} \delta J & \lambda \delta & 0 \\
0 & -\lambda \delta J & \delta F^{t}-F \delta & \delta G^{t} \\
0 & 0 & -G \delta & 0
\end{array}\right)
$$

is flat for any $\lambda \in \mathbb{C}$. Here $\delta=\operatorname{diag}\left(d x_{1}, \ldots, d x_{n+1}\right)$ and the matrices are partitioned into blocks with sizes $(1, n+1, n+1, k-n-1)$.

Since

$$
\left(\begin{array}{cc}
\delta F^{t}-F \delta & \delta G^{t} \\
-G \delta & 0
\end{array}\right)
$$

is flat in (4.1), there exists a map $B \in O(k)$ such that

$$
B d B^{-1}=\left(\begin{array}{cc}
\delta F^{t}-F \delta & \delta G^{t} \\
-G \delta & 0
\end{array}\right) .
$$

Put $B=\left(\begin{array}{l}B_{1} \\ B_{2}\end{array}\right)$, where $B_{1} \in \mathcal{M}_{(n+1) \times k}$. Taking a gauge transformation on $\theta_{\lambda}$ by $g=\left(\begin{array}{ccc}1 & 0 & 0 \\ 0 & I_{n+1} & 0 \\ 0 & 0 & B^{-1}\end{array}\right)$ gives

$$
g * \theta_{\lambda}=\left(\begin{array}{ccc}
0 & -c b^{t} \delta J & 0 \\
\delta b & \delta F-J F^{t} \delta J & \lambda \delta B_{1} \\
0 & -\lambda B_{1}^{t} \delta J & 0
\end{array}\right) .
$$

We also say $\left(b, F, B_{1}\right)$ is an associated solution to $(b, F, G)$ of the Lorentzian Grassmannian system when $g * \theta_{\lambda}$ is flat for any $\lambda$. Notice that the flatness of $g * \theta_{\lambda}$ is exactly the equations (2.3). From this fact, we get the following proposition [4].

Proposition 4.2. Assume $k \geq n+1$. Let $X: N^{n, 1}(c) \longrightarrow N^{n+k, 1}(c)$ be a nondegenerate local isometric immersion with a flat normal bundle, and for a local parallel normal frame $e_{\alpha}$ the first and second fundamental forms are given by

$$
I=\sum_{i=1}^{n+1} \epsilon_{i} b_{i}^{2} d x_{i}^{2}, \quad I I=\sum_{i=1}^{n+1} \sum_{r=1}^{k} \epsilon_{i} b_{i r} b_{i} d x_{i}^{2} \otimes e_{n+1+r}
$$

Then for $b=\left(b_{1}, \ldots, b_{n+1}\right)^{t}$ and $B_{1}=\left(b_{i r}\right),\left(b, F, B_{1}\right)$ is an associated solution to the Lorentzian Grassmannian system.

Conversely, any associated solution $\left(b, F, B_{1}\right)$ to the Lorentzian Grassmannian system gives rise to an immersion $X$ as above.

Now, we construct two kind of dressing actions ([10]) on the space of solutions of the Lorentzian Grassmannian system, which turns out to be a Ribaucour transformation of $N^{n, 1}(c)$ in $N^{n+k, 1}(c)$. This kind of action was used in [1] to get Ribaucour transformations of $N^{n}(c)$ in $N^{n+k}(c)$. 
Recall $J=\operatorname{diag}(-1,1, \ldots, 1), J_{c}=\operatorname{diag}(c,-1,1, \ldots, 1)$. The reality conditions for the Lorentzian Grassmannian system are

$$
\left\{\begin{array}{l}
g(\lambda) J_{c} g(\lambda)^{t}=J_{c}, \\
\left(\begin{array}{ccc}
1 & 0 & 0 \\
0 & I_{n+1} & 0 \\
0 & 0 & -I_{k}
\end{array}\right) g(\lambda)\left(\begin{array}{ccc}
1 & 0 & 0 \\
0 & I_{n+1} & 0 \\
0 & 0 & -I_{k}
\end{array}\right)=g(-\lambda), \\
g(\bar{\lambda})=g(\lambda) .
\end{array}\right.
$$

A trivialization $E_{\lambda}$ of $\theta_{\lambda}$, which means $E_{\lambda}^{-1} d E_{\lambda}=\theta_{\lambda}$, satisfies the reality conditions (4.4) and is holomorphic for any $\lambda \in \mathbb{C}$.

For $z=\left(z_{0}, z_{1}, \ldots, z_{n+1}\right)^{t} \in \mathbb{R}^{n+2}$ and $w=\left(w_{1}, \ldots, w_{k}\right)^{t} \in \mathbb{R}^{k}$ such that $\|z\|_{n+1, c}^{2}=z^{t} J_{c} z=1$ and $\|w\|_{k}^{2}=w^{t} w=1$, let

$$
\pi=\frac{1}{2}\left(\begin{array}{cc}
z z^{t} J_{c} & -i z w^{t} \\
i w z^{t} J_{c} & w w^{t}
\end{array}\right)
$$

Define $q_{s, \pi}(\lambda)$ for $s \in \mathbb{R}$ with $s \neq 0$ and $\lambda \in \mathbb{C}$ by

$$
q_{s, \pi}(\lambda)^{t}=\left(\pi+\frac{\lambda+i s}{\lambda-i s}(1-\pi)\right)\left(\bar{\pi}+\frac{\lambda-i s}{\lambda+i s}(1-\bar{\pi})\right) .
$$

Then it is easy to see that $q_{s, \pi}(\lambda)$ is invertible, satisfies the reality conditions (4.4), is holomorphic at $\lambda=\infty$, and

$$
q_{s, \pi}(\lambda)=q_{s, \pi}(-\lambda)^{-1}=I+\frac{2 s}{\lambda^{2}+s^{2}}\left(\begin{array}{cc}
-s J_{c} z z^{t} & -\lambda J_{c} z w^{t} \\
\lambda w z^{t} & -s w w^{t}
\end{array}\right)
$$

Lemma 4.3. Let $\left(\begin{array}{c}\tilde{z} \\ i \tilde{w}\end{array}\right)=E_{-i s}(x)^{t}\left(\begin{array}{c}z \\ i w\end{array}\right)$. Then

(i) $\tilde{z} \in \mathbb{R}^{n+2}$ and $\tilde{w} \in \mathbb{R}^{k}$,

(ii) $\|\tilde{z}\|_{n+1, c}^{2}=\|\tilde{w}\|_{k}^{2}$,

(iii) $\left(\tilde{z}_{0}\right)_{x_{i}}=b_{i} \tilde{z}_{i}$,

(iv) $\left(\tilde{z}_{j}\right)_{x_{i}}=\tilde{z}_{i} f_{i j}$ for $i \neq j$,

(v) $\left[\left(B^{t} \tilde{w}\right)_{r}\right]_{x_{i}}=s \tilde{z}_{i} b_{i r}$.

Proof. (i) follows easily from the reality conditions (4.4) of $q_{s, \pi}(\lambda)$. From

$$
\begin{aligned}
\|\tilde{z}\|_{n+1, c}^{2}-\|\tilde{w}\|_{k}^{2} & =\left(E_{-i s}(x)^{t}\left(\begin{array}{c}
z \\
i w
\end{array}\right)\right)^{t} J_{c}\left(E_{-i s}(x)^{t}\left(\begin{array}{c}
z \\
i w
\end{array}\right)\right) \\
& =\left(\begin{array}{c}
z \\
i w
\end{array}\right)^{t} J_{c}\left(\begin{array}{c}
z \\
i w
\end{array}\right) \\
& =\|z\|_{n+1, c}^{2}-\|w\|_{k}^{2} \\
& =0
\end{aligned}
$$


we have $\|\tilde{z}\|_{n+1, c}^{2}=\|\tilde{w}\|_{k}^{2}$. To prove (iii), (iv) and (v), take $d$ on the hypothesis, then

$$
\begin{aligned}
d\left(\begin{array}{c}
\tilde{z} \\
B^{t} \tilde{w}
\end{array}\right) & =d\left[g\left(\begin{array}{c}
\tilde{z} \\
i \tilde{w}
\end{array}\right)\right] \quad \text { for } \quad g=\left(\begin{array}{cc}
I & 0 \\
0 & B^{t}
\end{array}\right) \\
& =d g \cdot g^{t}\left(\begin{array}{c}
\tilde{z} \\
i B^{t} \tilde{w}
\end{array}\right)+g \cdot d E_{-i s}(x)^{t} \cdot\left(E_{-i s}(x)^{t}\right)^{-1}\left(\begin{array}{c}
\tilde{z} \\
i \tilde{w}
\end{array}\right) \\
& =\left(d g \cdot g^{t}+g \theta_{-i s}^{t} g^{t}\right)\left(\begin{array}{c}
\tilde{z} \\
i B^{t} \tilde{w}
\end{array}\right) \\
& =\left(g * \theta_{-i s}\right)^{t}\left(\begin{array}{c}
\tilde{z} \\
i B^{t} \tilde{w}
\end{array}\right) \text { by }(4.3) .
\end{aligned}
$$

Thus the results follow from

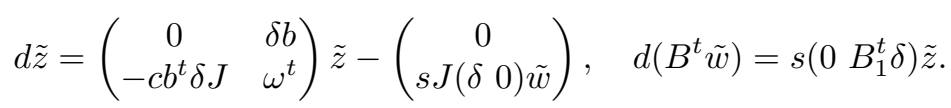

Put $\hat{z}=\tilde{z} /\|\tilde{z}\|_{n+1, c}, \hat{w}=\tilde{w} /\|\tilde{w}\|_{k}$ and $\tilde{\pi}=\frac{1}{2}\left(\begin{array}{cc}\hat{z} \hat{z}^{t} J_{c} & -i \hat{z} \hat{w}^{t} \\ i \tilde{w} \hat{z}^{t} J_{c} & \hat{w} \hat{w}^{t}\end{array}\right)$. It is easy to show that $q_{s, \pi}(\lambda) E_{\lambda} q_{s, \tilde{\pi}}(\lambda)^{-1}$ is holomorphic in $\lambda \in \mathbb{C}$ by residue calculations at $\lambda= \pm i s$. Let $\tilde{E}_{\lambda}=E_{\lambda} q_{s, \tilde{\pi}}(\lambda)^{-1}$, that is,

$$
\tilde{E}_{\lambda}=E_{\lambda}\left(\begin{array}{cc}
I-\frac{2 s^{2}}{\lambda^{2}+s^{2}} J_{c} \hat{z} \hat{z}^{t} & \frac{2 s \lambda}{\lambda^{2}+s^{2}} J_{c} \hat{z} \hat{w}^{t} \\
-\frac{2 s \lambda}{\lambda^{2}+s^{2}} \hat{w} \hat{z}^{t} & I-\frac{2 s^{2}}{\lambda^{2}+s^{2}} \hat{w} \hat{w}^{t}
\end{array}\right) .
$$

Now, from $E_{\lambda}^{-1} d E_{\lambda}=\sum_{i=1}^{n+1}\left(a_{i} \lambda+\left[a_{i}, v\right]\right) d x_{i}$ and $q_{s, \tilde{\pi}}(\lambda)$ is holomorphic at $\lambda=\infty$, we can prove by direct calculation that $\tilde{\theta}_{\lambda}=\tilde{E}_{\lambda}^{-1} d \tilde{E}_{\lambda}$ is of the form

$$
\tilde{\theta}_{\lambda}=\sum_{i=1}^{n+1}\left(a_{i} \lambda+\left[a_{i}, \tilde{v}\right]\right) d x_{i}
$$

and when we write

$$
v=\left(\begin{array}{cc}
0 & -J_{c} \xi^{t} \\
\xi & 0
\end{array}\right) \quad \text { and } \quad \tilde{v}=\left(\begin{array}{cc}
0 & -J_{c} \tilde{\xi}^{t} \\
\tilde{\xi} & 0
\end{array}\right)
$$

we obtain

$$
\tilde{\xi}=\xi-\frac{2}{s}\left(\hat{w} \hat{z}^{t}\right)_{*}
$$

where $\left.\left(\begin{array}{cc}0 & -\left(J_{c} \xi^{t}\right. \\ \xi_{*} & 0\end{array}\right)_{*}\right)$ is the orthogonal projection of $\left(\begin{array}{c}\eta \\ \xi \\ \xi\end{array}\right)$ onto $\mathcal{P} \cap J_{c} \xi^{t}$.

By the above argument, we get

Theorem 4.4. Let $(F, G, b)$ be a solution of the Lorentzian Grassmannian system whose corresponding one parameter family of flat connections is $\theta_{\lambda}$ in 
(4.1). Then we have a new solution $(\tilde{F}, \tilde{G}, \tilde{b})$ corresponding to $\tilde{\theta}_{\lambda}$ in $(4.7)$. In particular,

$$
\left(\begin{array}{cc}
\tilde{b} & \tilde{F} \\
0 & \tilde{G}
\end{array}\right)=\left(\begin{array}{cc}
b & F \\
0 & G
\end{array}\right)-\frac{2}{s}\left(\hat{w} \hat{z}^{t}\right)_{*}
$$

where $\left(c_{i j}\right)_{*}$ means $c_{i, i+1}=0$.

Hence we obtain a new solution $\tilde{X}$ corresponding to $(\tilde{F}, \tilde{G}, \tilde{b})$ from a given immersion $X$ for $(F, G, b)$. We will investigate on how $X$ and $\tilde{X}$ are related geometrically. Let $E_{\lambda}$ and $\tilde{E}_{\lambda}$ be trivializations corresponding to $\theta_{\lambda}$ and $\tilde{\theta}_{\lambda}$ in (4.1) and (4.7), respectively. Write

$$
E_{0}(x)=\left(\begin{array}{cc}
A(x) & 0 \\
0 & B(x)^{-1}
\end{array}\right), \quad \tilde{E}_{0}(x)=\left(\begin{array}{cc}
\tilde{A}(x) & 0 \\
0 & \tilde{B}(x)^{-1}
\end{array}\right) .
$$

From (4.6), we have

$$
\tilde{A}=A\left(I-2 J_{c} \hat{z} \hat{z}^{t}\right), \quad \tilde{B}^{-1}=B^{-1}\left(I-2 \hat{w} \hat{w}^{t}\right) .
$$

Put

$$
E_{\lambda}^{I}=E_{\lambda}\left(\begin{array}{cc}
I_{n+2} & 0 \\
0 & B
\end{array}\right), \quad \tilde{E}_{\lambda}^{I}=\tilde{E}_{\lambda}\left(\begin{array}{cc}
I_{n+2} & 0 \\
0 & \tilde{B}
\end{array}\right) .
$$

In fact, if we write $E_{1}^{I}=\left(X, e_{1}, \ldots, e_{n+1}, e_{n+2}, \ldots, e_{n+k+1}\right)$, then $X$ is the immersion of $N^{n, 1}(c)$ into $N^{n+k, 1}(c), e_{i}(1 \leq i \leq n+1)$ are a tangent frame and $e_{\alpha}(n+2 \leq \alpha \leq n+k+1)$ are a parallel normal frame to $X$. By a direct calculation, these trivializations are related by

$$
\tilde{E}_{1}^{I}=E_{1}^{I}\left(I-\frac{2}{1+s^{2}}\left(\begin{array}{c}
s J_{c} \hat{z} \\
B^{t} \hat{w}
\end{array}\right)\left(\begin{array}{ll}
s \hat{z}^{t} & \hat{w}^{t} B
\end{array}\right)\right) .
$$

Now we obtain

Theorem 4.5. Suppose $X: N^{n, 1}(c) \rightarrow N^{n+k, 1}(c)$ is a nondegenerate local isometric immersion with flat normal bundle. Then the $q_{s, \pi}(\lambda)$ action on $X$ gives rise to a new immersion $\tilde{X}: N^{n, 1}(c) \rightarrow N^{n+k, 1}(c)$ of the same kind, and $X$ and $\tilde{X}$ are in a Ribaucour transformation in tangentially spacelike direction.

Proof. Let $E_{\lambda}$ be a trivialization of $\theta_{\lambda}$ in (4.1) and $\tilde{E}_{\lambda}=E_{\lambda} q_{s, \tilde{\pi}(x)}(\lambda)^{-1}$. Write

$$
\begin{aligned}
& E_{1}^{I}=\left(X, e_{1}, \ldots, e_{n+1}, e_{n+2}, \ldots, e_{n+k+1}\right), \\
& \tilde{E}_{1}^{I}=\left(\tilde{X}, \tilde{e}_{1}, \ldots, \tilde{e}_{n+1}, \tilde{e}_{n+2}, \ldots, \tilde{e}_{n+k+1}\right) .
\end{aligned}
$$

By (4.10), we have

$$
\tilde{E}_{1}^{I}=E_{1}^{I}\left(I-2\left(\begin{array}{c}
\cos \rho J_{c} \hat{z} \\
\sin \rho B^{t} \hat{w}
\end{array}\right)\left(\begin{array}{ll}
\cos \rho \hat{z}^{t} & \left.\sin \rho \hat{w}^{t} B\right)
\end{array}\right),\right.
$$

where $s=\cot \rho$. Put

$$
\tilde{\gamma}=\cos \rho \tilde{z}(x)^{t}, \quad \tilde{\beta}=\sin \rho \tilde{w}(x)^{t} B, \quad \eta=E_{1}^{I}\left(\begin{array}{c}
\cos \rho J_{c} \hat{z}(x) \\
\sin \rho B^{t} \hat{w}(x)
\end{array}\right) .
$$


Then it follows from (4.11) that

$$
\begin{array}{rlr}
\tilde{X} & =X-\frac{2 \tilde{\gamma}_{0}}{\|\tilde{w}\|} \eta, & \\
\tilde{e}_{i} & =e_{i}-\frac{2 \tilde{\tilde{\gamma}_{i}}}{\|\tilde{w}\|} \eta, \quad(1 \leq i \leq n+1), \\
\tilde{e}_{\alpha} & =e_{\alpha}-\frac{2 \tilde{\beta}_{\alpha-n-1}}{\|\tilde{w}\|} \eta, \quad(\alpha \geq n+2) .
\end{array}
$$

Hence $X+r_{i} e_{i}=\tilde{X}+r_{i} \tilde{e}_{i}$ and $X+r_{\alpha} e_{\alpha}=\tilde{X}+r_{\alpha} \tilde{e}_{\alpha}$ for $r_{i}=-\tilde{\gamma}_{0} / \tilde{\gamma}_{i}$ and $r_{\alpha}=-\tilde{\gamma}_{0} / \tilde{\beta}_{\alpha-n-1}$. Therefore, $X$ and $\tilde{X}$ are in a Ribaucour transformation.

If we write $(4.13)$ in terms of $\tilde{\gamma}$ and $\tilde{\beta}$, then

$$
\tilde{X}=X-\frac{2 \sin \rho \tilde{\gamma}_{0}}{\|\tilde{\beta}\|^{2}}\left(c \tilde{\gamma}_{0} X+\sum_{i} \epsilon_{i} \tilde{\gamma}_{i} e_{i}+\sum_{r} \tilde{\beta}_{r} e_{n+1+r}\right)
$$

Theorem 4.6. Any Ribaucour transformation $\tilde{X}$ in tangentially spacelike direction of a nondegenerate isometric immersion $X: N^{n, 1}(c) \longrightarrow N^{n+k, 1}(c)$ with flat normal bundle can be obtained by some $q_{s, \pi}(\lambda)$ action on $X$.

Proof. Let $\gamma$ and $\beta$ be given as in Theorem 3.2. According to Lemma 4.3 and (3.6), $(\gamma, \beta)$ and $(\tilde{\gamma}, \tilde{\beta})$ satisfy the same differential equations. Thus if we take $z=\sec \rho \gamma(0)^{t}$ and $w=\csc \rho \beta(0)^{t} B(0)^{t}$, then they have the same initial values and hence they are the same.

Now, we construct another kind of an action. Take $z=\left(z_{0}, z_{1}, \ldots, z_{n+1}\right)^{t} \in$ $\mathbb{R}^{n+2}$ and $w=\left(w_{1}, \ldots, w_{k}\right)^{t} \in \mathbb{R}^{k}$ so that $\|z\|_{n+1, c}^{2}=z^{t} J_{c} z=-1$ and $\|w\|_{k}^{2}=$ $w^{t} w=1$. Let

$$
\pi=\frac{1}{2}\left(\begin{array}{cc}
-z z^{t} J_{c} & z w^{t} \\
-w z^{t} J_{c} & w w^{t}
\end{array}\right) \quad \text { and } \quad \pi^{\prime}=\frac{1}{2}\left(\begin{array}{cc}
-z z^{t} J_{c} & -z w^{t} \\
w z^{t} J_{c} & w w^{t}
\end{array}\right) .
$$

Define $p_{s, \pi}(\lambda)$ for $s \in \mathbb{R}$ with $s \neq 0$ and $\lambda \in \mathbb{C}$ by

$$
p_{s, \pi}(\lambda)^{t}=\left(\pi+\frac{\lambda+s}{\lambda-s}(1-\pi)\right)\left(\pi^{\prime}+\frac{\lambda-s}{\lambda+s}\left(1-\pi^{\prime}\right)\right) .
$$

Then it is easy to see that $p_{s, \pi}(\lambda)$ is invertible, satisfies the reality conditions (4.4), is holomorphic at $\lambda=\infty$. Also, $\pi \pi^{\prime}=\pi^{\prime} \pi=0$ and

$$
p_{s, \pi}(\lambda)=p_{s, \pi}(-\lambda)^{-1}=I+\frac{2 s}{\lambda^{2}-s^{2}}\left(\begin{array}{cc}
-s J_{c} z z^{t} & \lambda J_{c} z w^{t} \\
-\lambda w z^{t} & s w w^{t}
\end{array}\right) .
$$

As does for $q_{s, \pi}(\lambda)$, the following holds for $p_{s, \pi}(\lambda)$ by a direct computation.

Lemma 4.7. Let $\left(\begin{array}{c}\tilde{z} \\ \tilde{w}\end{array}\right)=E_{-s}(x)^{t}\left(\begin{array}{c}z \\ w\end{array}\right)$. Then

(i) $\tilde{z} \in \mathbb{R}^{n+2}$ and $\tilde{w} \in \mathbb{R}^{k}$,

(ii) $\|\tilde{z}\|_{n+1, c}^{2}=-\|\tilde{w}\|_{k}^{2}$,

(iii) $\left(\tilde{z}_{0}\right)_{x_{i}}=b_{i} \tilde{z}_{i}$,

(iv) $\left(\tilde{z}_{j}\right)_{x_{i}}=\tilde{z}_{i} f_{i j}$ for $i \neq j$,

(v) $\left[\left(B^{t} \tilde{w}\right)_{r}\right]_{x_{i}}=s \tilde{z}_{i} b_{i r}$. 
Again, put $\hat{z}=\tilde{z} /\|\tilde{w}\|_{k}, \hat{w}=\tilde{w} /\|\tilde{w}\|_{k}, \tilde{E}_{\lambda}=E_{\lambda} p_{s, \tilde{\pi}}(\lambda)^{-1}$. Then,

$$
\tilde{E}_{\lambda}=E_{\lambda}\left(\begin{array}{cc}
I-\frac{2 s^{2}}{\lambda^{2}-s^{2}} J_{c} \hat{z} \hat{z}^{t} & -\frac{2 s \lambda}{\lambda^{2}-s^{2}} J_{c} \hat{z} \hat{w}^{t} \\
\frac{2 s \lambda}{\lambda^{2}-s^{2}} \hat{w} \hat{z}^{t} & I-\frac{2 s^{2}}{\lambda^{2}-s^{2}} \hat{w} \hat{w}^{t}
\end{array}\right) .
$$

Similarly to (4.8) and (4.9), we get frames

$$
\tilde{E}_{1}^{I}=E_{1}^{I}\left(I-\frac{2}{1-s^{2}}\left(\begin{array}{c}
s J_{c} \hat{z} \\
B^{t} \hat{w}
\end{array}\right)\left(\begin{array}{ll}
s \hat{z}^{t} & \hat{w}^{t} B
\end{array}\right)\right) .
$$

Summarizing the above argument, we get the following theorems, which we omit the details of the proofs.

Theorem 4.8. Suppose $X: N^{n, 1}(c) \rightarrow N^{n+k, 1}(c)$ is a nondegenerate local isometric immersion with flat normal bundle. Then the $p_{s, \pi}(\lambda)$ action on $X$ gives rise to a new immersion $\tilde{X}: N^{n, 1}(c) \rightarrow N^{n+k, 1}(c)$ of the same kind, and $X$ and $\tilde{X}$ are in a Ribaucour transformation in tangentially timelike direction. In particular, the immersions, tangent and normal frames are related by

$$
\begin{array}{rlr}
\tilde{X} & =X-\frac{2 \tilde{\gamma}_{0}}{\|\tilde{w}\|} \eta, & \\
\tilde{e}_{i} & =e_{i}-\frac{2 \tilde{\tilde{n}}}{\|\tilde{w}\|} \eta, & (1 \leq i \leq n+1), \\
\tilde{e}_{\alpha} & =e_{\alpha}-\frac{2 \tilde{\beta}_{\alpha-n-1}}{\|\tilde{w}\|} \eta, & (\alpha \geq n+2),
\end{array}
$$

where when $0<s<1$,

$$
s=\tanh \rho, \tilde{\gamma}=\sinh \rho \tilde{z}(x)^{t}, \tilde{\beta}=\cosh \rho \tilde{w}(x)^{t} B, \eta=E_{1}^{I}\left(\begin{array}{c}
\sinh \rho J_{c} \hat{z}(x) \\
\cosh \rho B^{t} \hat{w}(x)
\end{array}\right),
$$

and when $s>1$,

$$
s=\operatorname{coth} \rho, \tilde{\gamma}=\cosh \rho \tilde{z}(x)^{t}, \tilde{\beta}=\sinh \rho \tilde{w}(x)^{t} B, \eta=-E_{1}^{I}\left(\begin{array}{c}
\cosh \rho J_{c} \hat{z}(x) \\
\sinh \rho B^{t} \hat{w}(x)
\end{array}\right) .
$$

Theorem 4.9. Any Ribaucour transformation $\tilde{X}$ in tangentially timelike direction of a nondegenerate isometric immersion $X: N^{n, 1}(c) \longrightarrow N^{n+k, 1}(c)$ with flat normal bundle can be obtained by some $p_{s, \pi}(\lambda)$ action on $X$. In particular, $\tilde{X}$ is of the form

$$
\tilde{X}=X-\frac{2 \cosh \rho \tilde{\gamma}_{0}}{\|\tilde{\beta}\|^{2}}\left(c \tilde{\gamma}_{0} X+\sum_{i} \epsilon_{i} \tilde{\gamma}_{i} e_{i}+\sum_{r} \tilde{\beta}_{r} e_{n+1+r}\right)
$$

or

$$
\tilde{X}=X+\frac{2 \sinh \rho \tilde{\gamma}_{0}}{\|\tilde{\beta}\|^{2}}\left(c \tilde{\gamma}_{0} X+\sum_{i} \epsilon_{i} \tilde{\gamma}_{i} e_{i}+\sum_{r} \tilde{\beta}_{r} e_{n+1+r}\right)
$$

where $s=\tanh \rho$ or $s=\operatorname{coth} \rho$. 


\section{References}

[1] M. Brück, X. Du, J. Park, and C. L. Terng, The submanifold geometries associated to Grassmannian systems, Mem. Amer. Math. Soc. 155 (2002), no. 735, viii+95 pp.

[2] M. Dajczer and R. Tojeiro, An extension of the classical Ribaucour transformation, Proc. London Math. Soc. (3) 85 (2002), no. 1, 211-232.

[3] L. P. Eisenhart, A Treatise on The Differential Geometry of Curves and Surfaces, Dover Publications, Inc., New York 1960.

[4] J. Park, Lorentzian submanifolds in Lorentzian space form with the same constant curvatures, Geom. Dedicata 108 (2004), 93-104.

[5] _ Lorentzian surfaces with constant curvatures and transformations in the 3dimensional Lorentzian space, J. Korean Math. Soc. 45 (2008), no. 1, 41-61.

[6] K. Tenenblat, Bäcklund's theorem for submanifolds of space forms and a generalized wave equation, Bol. Soc. Brasil. Mat. 16 (1985), no. 2, 69-94.

[7] K. Tenenblat and C. L. Terng, Bäcklund's theorem for $n$-dimensional submanifolds of $\mathbb{R}^{2 n-1}$, Ann. of Math. (2) 111 (1980), no. 3, 477-490.

[8] C. L. Terng, A higher dimension generalization of the sine-Gordon equation and its soliton theory, Ann. of Math. (2) 111 (1980), no. 3, 491-510.

[9] _ Soliton equations and differential geometry, J. Differential Geom. 45 (1997), no. $2,407-445$.

[10] K. Uhlenbeck, Harmonic maps into Lie groups: classical solutions of the chiral model, J. Differential Geom. 30 (1989), no. 1, 1-50.

Department of Mathematics

DONGGUK UNIVERSITY

SeOul 100-715, Korea

E-mail address: jpark@dgu.ac.kr 